\section{Validação de escalas psicossociais para mudança do consumo de frutas, legumes e verduras}

\author{
Validation of psychosocial scales for change \\ in the consumption of fruits and vegetables \\ Validación de escalas psicosociales para el
cambio en el consumo de frutas y verduras
}

\begin{abstract}
The objective of this study was to translate, adapt, and test the psychometric properties of psychosocial scales on fruits and vegetable consumption. A multidimensional questionnaire based on constructs of Social Cognitive Theory and the Transtheoretical Model was administered to 717 university students. Validation procedures included conceptual, item, semantic, operational, measurement, and functional equivalence. Exploratory factor analysis and temporal stability were performed using SPSS (17.0). Small differences between the translated versions were observed, and a few adjustments were made. The explained variance ranged from $57.3 \%$ to $63.2 \%$, and alpha values ranged from 0.77 to 0.88 , showing good internal consistency. Average temporal stability was good, and all constructs correlated with the stages of behavior change $(p<0.05)$. All validation steps were satisfactory and deemed appropriate for application in the population.
\end{abstract}

Food Consumption; Nutrition; Questionnaires
Rafael Miranda Tassitano 1

Poliana Coelho Cabral 2

Giselia Alves Pontes da Silva 2

\section{Resumo}

O objetivo foi traduzir, adaptar e testar as propriedades psicométricas de mediadores psicossociais para o consumo de frutas, legumes e verduras. Um questionário multidimensional baseado nos construtos da Teoria Cognitiva Social e do Modelo Transteorético foi administrado em 717 estudantes universitários. Os procedimentos de validação foram: equivalência conceitual, de itens, semântica, operacional, de mensuração e funcional. Análise fatorial exploratória e de estabilidade temporal foram realizadas usando-se o programa SPSS (17.0). Pequenas diferenças entre as versões traduzidas foram observadas e poucas adaptações foram realizadas. A variância explicada observada ficou entre $57,3 \%$ e 63,2\%, e os valores de $\alpha$ variou de 0,77 a 0,88, apresentando boa consistência interna. A estabilidade temporal média foi boa e todos os construtos se correlacionaram ao estágio de mudança de comportamento $(p<0,05)$. Todas as etapas de validação foram satisfatórias e consideradas adequadas para aplicação na população.

Consumo de Alimentos; Nutrição;

Questionários 


\section{Introdução}

O consumo regular de frutas, legumes e verduras faz parte de qualquer estratégia de promoção da saúde devido às evidências acumuladas nas últimas décadas 1,2,3. Apesar dos reconhecidos benefícios e dos esforços das políticas públicas para promover uma alimentação saudável, o consumo desses alimentos, na grande maioria dos países e no Brasil, permanece baixo ${ }^{3}$. Teorias do comportamento têm sido propostas para explicar como o indivíduo estabelece e mantém um determinado comportamento saudável 4 . Intervenções com o objetivo de aumentar o consumo baseadas em estratégia de modificação de fatores psicossociais apresentam maior impacto, quando comparadas a intervenções que não utilizaram esta estratégia, independentemente do contexto e da faixa etária 5 .

Entretanto, estudos de revisão sistemática têm indicado não existir consenso de quais construtos e/ou modelos teóricos melhor explicam o comportamento 6,7. Sabe-se que a autoeficácia e o suporte social são fortes preditores ${ }^{6}$, mas quando analisados trabalhos que utilizaram todos os construtos de uma teoria com o objetivo de estabelecer um modelo preditivo, os modelos explicam entre $23 \%$ e $34 \%$ do consumo 7 . Essa variabilidade de resultados é ressaltada em ambos os artigos de revisão sistemática que identificam a dificuldade de fazer comparações diretas entre os estudos, devido a limitações metodológicas, principalmente em relação à avaliação do consumo alimentar e à validação dos construtos psicossociais 8,9 .

Grupo de pesquisadores da Universidade de San Diego (Estados Unidos) tem desenvolvido trabalhos sobre a mudança de comportamentos de saúde e estilos de vida com base na Teoria Cognitiva Social 10 e no Modelo Transteorético ${ }^{11}$. Assim, desenvolveram, validaram e utilizaram em estudos com diferentes delineamentos epidemiológicos os construtos dessas teorias para diversos comportamentos de saúde $12,13,14,15,16,17,18,19$. No Brasil, não foram localizados estudos de validação de escalas psicossociais para a mudança de comportamento do consumo de frutas, legumes e verduras. Sendo assim, o presente trabalho tem como objetivo traduzir, adaptar e testar as propriedades psicométricas dos construtos psicossociais dessas teorias.

\section{Materiais e métodos}

Trata-se de um estudo de validação de instrumento com estudantes da Universidade Federal Rural de Pernambuco (UFRPE). O protocolo de pesquisa foi aprovado pelo Comitê de Ética da Universidade Federal de Pernambuco (UFPE) sob o protocolo no 313/10. Além disso, a validação foi autorizada pelos autores do instrumento original.

Apesar de não existir um tamanho mínimo nem máximo de amostra para estudos de validação, a literatura recomenda uma proporção de 1:5 e 1:10 de cada item a ser validado por indivíduo 20. Entretanto, optou-se por estimar e dimensionar uma amostra que também fosse representativa da população do estudo, considerando o curso, turno e sexo. A coleta de dados ocorreu entre outubro e novembro de 2010. Um total de oito entrevistadores, previamente capacitados, conduziu as entrevistas de forma individual com duração de 25 a 35 minutos.

O procedimento de validação baseado na proposta de Reichenheim \& Moraes 21, é composto por seis etapas de equivalência: (a) conceitual; (b) de itens; (c) semântica; (d) operacional; (e) de mensuração; (f) funcional. As duas primeiras etapas foram realizadas por um comitê formado pelos autores do artigo e tiveram como objetivo verificar conceitualmente a relevância do instrumento e dos itens que o compõem. Informações adicionais foram solicitadas aos pesquisadores de San Diego.

A equivalência semântica foi feita em cinco passos: (a) tradução; (b) retrotradução; (c) equivalência semântica das versões; (d) adaptação cultural; e (e) pré-testagem. A tradução do instrumento original da língua inglesa para a língua portuguesa foi feita independentemente por dois profissionais fluentes em inglês (T1 e T2). As duas versões foram retraduzidas por um professor de inglês nativo com fluência em português (RT1). Na avaliação semântica foi verificado o significado denotativo (correspondência literal entre as versões) e conotativo (uso da palavra com um significado diferente do original). Ao final, a versão foi aplicada a vinte estudantes, com o objetivo de avaliar a clareza e o grau de compreensão de cada questão. Para cada uma delas foi perguntado: “Você entendeu o que foi perguntado?", sendo as opções de resposta: não entendi nada; entendi só um pouco; entendi mais ou menos; entendi quase tudo, mas tive algumas dúvidas; entendi quase tudo; e entendi perfeitamente e não tenho dúvidas.

A avaliação do layout, as orientações de preenchimento e sobre os construtos, a sequência das perguntas, as opções de resposta e o modo de aplicação compuseram a equivalência operacional, e a verificação das propriedades psicométricas correspondeu à equivalência de mensuração. Nesse último procedimento foram adotados três enfoques: (a) avaliação de validade dimensional 
e adequação de itens; (b) avaliação de confiabilidade; e (c) avaliação de validade de construto.

$\mathrm{O}$ instrumento é composto por 42 questões divididas em sete módulos: frequência de consumo de frutas, legumes e verduras $(\mathrm{n}=1)$; estágio de mudança de comportamento $(n=1)$; estratégia de mudança $(\mathrm{n}=15)$; processo de tomada de decisão $(\mathrm{n}=10)$; autoeficácia $(\mathrm{n}=7)$; suporte dos pais $(n=4)$ e amigos $(n=3)$; e se gosta de consumir frutas, legumes e verduras $(n=1)$ $12,13,16,17,18,19$. Maiores informações podem ser encontradas na página de Internet http://www. paceproject.org/measures.html.

A medida de frequência alimentar é composta por uma questão que pergunta a quantidade de porções de frutas, legumes e verduras que o indivíduo consome habitualmente em um dia típico, sendo as respostas dadas em número de porções somadas. As orientações sobre definição de porção também estão apresentadas no enunciado do instrumento original. Entretanto, optou-se por aplicar um questionário de frequência alimentar (QFA) de frutas, legumes e verduras elaborado e validado no Brasil 22, pois evidências indicam que deve-se levar em consideração as circunstâncias da cultura local 23. A inclusão também teve como objetivo comparar as duas formas de mensuração. O QFA utilizado é composto por 11 itens de verduras e legumes e dez de frutas, e as opções de respostas dadas são em frequência semanal ou diária. É importante ressaltar que, para cada alimento, o instrumento indica o que representa uma porção 22 . Assim, o número de porção total consumida diariamente se deu pelo somatório de todas elas.

O estágio de mudança de comportamento foi verificado de acordo com a sugestão da literatura 11,24. Para evitar a indicação errada dos estágios, a questão está integrada à do consumo alimentar, por meio de um organograma. Sendo assim, aqueles indivíduos que não atendem às recomendações ( $<5$ porções diárias) 3 têm como opções de resposta: pré-contemplação, contemplação ou preparação. E aqueles que já atendem às recomendações têm como opções de resposta: ação e manutenção.

O construto de estratégia de mudança é composto por 15 itens sobre situações que os indivíduos podem incorporar para mudar o comportamento 12. São cinco opções de resposta em escala Likert: nunca, quase nunca, às vezes, frequentemente, e várias vezes. O construto do processo de mudança de comportamento refere-se a aspectos cognitivos e motivacionais, sendo composto por cinco questões relacionadas à percepção dos facilitadores e cinco sobre a percepção das barreiras ${ }^{12}$. As opções de respostas dadas em escala Likert são: não é importante, um pouco impor- tante, mais ou menos importante, bastante importante, e extremamente importante.

A autoeficácia está relacionada ao grau de confiança/crença com que o indivíduo é capaz de realizar ou adotar determinado comportamento diante diversas barreiras existentes. São sete itens situacionais e as opções de resposta também são dadas em escala Likert: tenho certeza que não, provavelmente não, indiferente/neutro, provavelmente sim, e tenho certeza que sim. O suporte dos amigos é um construto semelhante ao suporte familiar com as mesmas opções de resposta 12: nunca, um a dois dias, três a quatro dias, cinco a seis dias, e todos os dias. A última questão do instrumento está relacionada ao grau de concordância em relação ao gostar de consumir frutas, legumes e verduras, com opções de respostas em escala Likert: discordo fortemente, discordo em partes, nem concordo e nem discordo, concordo em partes, e concordo fortemente. Para a caracterização da amostra foram incluídas informações sobre sexo, idade, nível sócioeconômico, estado civil, local de residência, trabalho e turno de estudo.

Os dados foram tabulados no Epidata, versão 3.1 (Epidata Assoc., Odense, Dinamarca) e todas as análises realizadas no software SPSS 17.0 (SPSS Inc., Chicago, Estados Unidos), sendo dividida em cinco etapas: (a) verificação do tamanho da amostra; (b) correlação entre a medida de consumo alimentar do questionário original e o QFA; (c) análise fatorial exploratória; (d) fidedignidade; (e) correlação do estágio de mudança de comportamento com as variáveis psicossociais.

Todos os construtos foram testados em relação à adequação da amostra pelo índice de Kaiser-Meyer-Olkin (KMO), no qual a literatura sugere como suficientes os valores acima de 0,7020 . Foi utilizado o teste de esfericidade de Bartelett, com o objetivo de verificar se a matriz de correlação é uma matriz identidade, não podendo o valor ultrapassar um valor de $\mathrm{p}$ de 0,05 20. Além disso, procurou-se identificar a existência de multicolinearidade (correlação alta) e singularidade (correlação perfeita) entre as questões. Esse último procedimento foi adotado para identificar a existência de itens que poderiam ser suprimidos. Utilizou-se o teste $t$ de Student para comparar os resultados das medidas de consumo alimentar obtidos no questionário original com o QFA 22. Para essa análise foi considerado que o valor de $\mathrm{p}$ não pode ser $<0,05$.

Após essas verificações, realizou-se a análise fatorial exploratória, nos respectivos construtos, com o emprego da rotação oblíqua promax para verificar a carga de cada item da escala. Foram aceitos autovalores (eingenvalues) $\geq 1,0$, além 
dos itens com carga $>0,4$, para definir os fatores obtidos na análise. Nessa etapa observou-se a consistência interna determinada pelo coeficiente alfa de Chronbach, considerando como satisfatórios os valores de alfa $\geq 0,720$. Foi testada a fidedignidade por meio da estabilidade temporal (coeficiente de correlação intraclasse - CCI), sendo esta obtida pelo método teste-reteste, com intervalos de aplicação de 10 a 14 dias em uma subamostra selecionada de forma aleatória $(n=53)$. Os critérios propostos por Landis \& Koch 25 foram adotados para a interpretação do grau de concordância: (a) quase perfeita: 0,80 a 1,00; (b) substancial: 0,60 a 0,80; (c) moderada: 0,40 a 0,60 ; d) regular: 0,20 a 0,40 ; (d) discreta: zero a 0,20 . Por fim, foi utilizada a correlação de Pearson para verificar a relação do estágio de mudança de comportamento com os construtos, adotando um valor de $\mathrm{p}<0,05$.

\section{Resultados}

Um total de 4,2\% dos estudantes se recusou a participar, fazendo parte efetivamente do estudo 717 alunos (55,9\% mulheres), com idade média e respectivo desvio-padrão observado de 20,6 \pm 1,9. E em linhas gerais a amostra foi composta por estudantes solteiros(as) $(95,4 \%)$, da zona urbana $(97,8 \%)$, que não trabalham $(64,6 \%)$, classificados segundo o nível socioeconômico nas classes B e/ou C $(87,1 \%)$ e que estudavam no turno diurno $(67,5 \%)$.

Todos os procedimentos referentes à equivalência semântica apresentaram resultados satisfatórios. As traduções mostraram equivalências entre si e a retrotradução apresentou semelhança à versão original. Na pré-testagem com os alunos, as questões obtiveram em sua grande maioria um grau de entendimento 5 (entendi perfeitamente e não tenho dúvidas), sendo poucas as alterações e/ou adaptações realizadas. Em todas as questões em que "fruits \& vegetables" aparece, o termo foi traduzido e adaptado para "frutas, legumes e verduras”. Nas questões Q2, Q33 e Q37, o termo "encourages" foi traduzido pelo T1 como "encorajar” e pelo T2 como “incentivar”. Já o termo "reward", na Q5, foi traduzido pelo T1 como "prêmio" e pelo T2 como "recompensa". Em ambos os casos o comitê optou pelas traduções do T2. Houve apenas uma adaptação, que ocorreu na Q16, quando a palavra "kids" foi traduzida por "crianças" e posteriormente substituída por "estudantes".

No processo de equivalência operacional foi respeitado o layout, a ordem de apresentação dos construtos e dos itens. Apenas na forma de aplicação houve a necessidade de explicar e citar exemplos em relação à porção das frutas, legumes e verduras, com o objetivo de não fazer o estudante nem superestimar nem subestimar a frequência do consumo alimentar. Além disso, optou-se por atribuir destaque em negrito às unidades temporais e à unidade de medida da resposta nos enunciados dos construtos. $\mathrm{Na} \mathrm{Ta}-$ bela 1, apresentamos a versão original e a versão final traduzida e adaptada.

No processo de equivalência de mensuração foram observados valores satisfatórios tanto para o teste de adequação da amostra (KMO) quanto para o teste de esfericidade de Bartelett (Tabelas 2, 3, 4 e 5). As análises de singularidade e multicolinearidade indicaram os seguintes valores mínimos e máximos de correlação: estratégia de mudança de comportamento $(0,24$ e 0,66$)$; percepção dos facilitadores $(0,32$ e 0,61) e percepção das barreiras $(0,27$ e 0,61); autoeficácia $(0,32$ e 0,66$)$; suporte familiar $(0,43$ e 0,67$)$ e suporte dos amigos $(0,45$ e 0,68$)$. Todos os resultados foram adequados, sendo inicialmente mantidos todos os itens para a análise fatorial exploratória. Quando analisadas a medida obtida pelo QFA com a do questionário original, verificou-se uma diferença, porém não significativa. Pelo primeiro método observou-se um consumo médio diário de 2,38 de frutas, legumes e verduras, e no segundo o consumo médio diário foi de $2,28(\mathrm{p}=0,20)$.

$\mathrm{Na}$ análise dos componentes principais, o construto estratégia de mudança de comportamento identificou apenas um fator que explicou $59,1 \%$ da variância da escala, com boa consistência interna (alfa de 0,88 ). Apenas a questão 2 (" $E u$ tenho um amigo ou parente que me incentiva a comer mais frutas, legumes e verduras") apresentou um valor de fator abaixo de 0,54. Mesmo assim não foi retirado, visto que sua exclusão não aumentaria o valor de alfa, além disto, está acima do considerado satisfatório $(>0,40)$. Os valores de teste e reteste apresentaram sempre índices acima de 0,64 , sendo que em cinco itens os valores foram superiores a 0,80. A correlação com o estágio de mudança de comportamento também apresentou resultados significativos com um r superior a 0,50.

O construto processo de tomada de decisão identificou dois fatores que, juntos, explicaram $57,7 \%$ da variância da escala, ambos com boa consistência interna. O primeiro, denominado "percepção dos facilitadores" obteve apenas uma questão no limiar estabelecido para a manutenção do item na escala $(0,48)$. A retirada da questão aumentaria a consistência interna, mas quando observado o intervalo de confiança, os valores de teste e reteste e a correlação com o estágio de mudança de comportamento, decidiuse manter a questão. Além disso, a manutenção 
Tabela 1

Versão original e versão traduzida e adaptada dos construtos psicossociais de frutas, legumes e verduras.

\begin{tabular}{|c|c|c|}
\hline \multicolumn{2}{|c|}{ Versão original } & \multirow{2}{*}{$\begin{array}{l}\text { Versão traduzida e adaptada } \\
\text { Estratégias de mudança do consumo de frutas, legumes e verduras }\end{array}$} \\
\hline C1 & Fruits \& vegetable change strategies & \\
\hline E2 & $\begin{array}{l}\text { The following are activities, thoughts, and feelings people use to } \\
\text { help them change their fruit \& vegetable intake. Think of any similar } \\
\text { experiences you may be having or have had in the past month. Then } \\
\text { rate HOW OFTEN you do each of the following. }\end{array}$ & $\begin{array}{l}\text { A seguir, encontram-se atividades, pensamentos e sentimentos } \\
\text { que as pessoas usam para ajudá-las a mudar a ingestão de frutas, } \\
\text { legumes e verduras. Pense sobre experiências semelhantes pelas } \\
\text { quais você pode estar passando ou ter passado no último mês. } \\
\text { Depois, marque COM QUE FREQUÊNCIA você vivencia cada uma } \\
\text { delas. }\end{array}$ \\
\hline Q1 & I set goals to eat at least five serving or fruits \& vegetables a day. & $\begin{array}{l}\text { Eu estabeleço metas para comer pelo menos cinco porções de } \\
\text { frutas, legumes e verduras por dia. }\end{array}$ \\
\hline Q2 & $\begin{array}{l}\text { I have a friend or family member who encourages me to eat more fruits } \\
\text { \& vegetables. }\end{array}$ & $\begin{array}{l}\text { Eu tenho um amigo ou parente que me incentiva a comer mais } \\
\text { frutas, legumes e verduras. }\end{array}$ \\
\hline Q3 & I say positive things to myself about eating fruits \& vegetables. & $\begin{array}{l}\text { Eu digo coisas positivas para mim mesmo sobre comer frutas e } \\
\text { legumes. }\end{array}$ \\
\hline Q4 & I think about the benefits I will get from eating fruits \& vegetables. & $\begin{array}{l}\text { Eu penso sobre os benefícios que vou ter por comer frutas, legumes } \\
\text { e verduras. }\end{array}$ \\
\hline Q5 & $\begin{array}{l}\text { I reward myself for eating at least five servings of fruits \& vegetables a } \\
\text { day. }\end{array}$ & $\begin{array}{l}\text { Eu me recompenso por comer pelo menos cinco porções de frutas, } \\
\text { legumes e verduras. }\end{array}$ \\
\hline Q6 & I look for information about ways to eat more fruits \& vegetables. & $\begin{array}{l}\text { Eu procuro informações sobre maneiras de comer mais frutas, } \\
\text { legumes e verduras. }\end{array}$ \\
\hline Q7 & $\begin{array}{l}\text { When I'm not eating enough fruits \& vegetables, I tell myself I can get } \\
\text { right back on track eating fruits \& vegetables. }\end{array}$ & $\begin{array}{l}\text { Quando não estou comendo frutas, legumes e verduras o suficiente, } \\
\text { digo que logo posso recomeçar e voltar a comer a quantidade } \\
\text { suficiente novamente. }\end{array}$ \\
\hline Q8 & I put reminders around my home to eat fruits \& vegetables. & $\begin{array}{l}\text { Eu coloco recados pela casa me lembrando de comer mais frutas, } \\
\text { legumes e verduras. }\end{array}$ \\
\hline Q9 & I keep track of the number of fruits \& vegetables I eat. & $\begin{array}{l}\text { Eu presto atenção à quantidade de frutas, legumes e verduras que } \\
\text { como. }\end{array}$ \\
\hline Q10 & $\begin{array}{l}\text { I think about how my surroundings affect the amount of fruits \& } \\
\text { vegetable I eat. (Surroundings are things like fast food restaurants and } \\
\text { pre-packaged foods in stores. }\end{array}$ & $\begin{array}{l}\text { Eu penso sobre como meu ambiente afeta a quantidade de frutas, } \\
\text { legumes e verduras que eu como (ambientes são coisas como } \\
\text { restaurantes fast-food e comidas congeladas). }\end{array}$ \\
\hline Q11 & $\begin{array}{l}\text { I find ways to get around the things that get in the way of eating fruits } \\
\text { \& vegetables. }\end{array}$ & $\begin{array}{l}\text { Eu encontro formas de resolver coisas que me atrapalhem comer } \\
\text { frutas, legumes e verduras. }\end{array}$ \\
\hline Q12 & I do things to make the eating fruits \& vegetables more enjoyable. & $\begin{array}{l}\text { Eu faço coisas para tornar o fato de comer frutas, legumes e verduras } \\
\text { mais agradável. }\end{array}$ \\
\hline Q13 & $\begin{array}{l}\text { I try to think more about the benefits of eating fruits \& vegetables and } \\
\text { less about the hassles of healthy eating. }\end{array}$ & $\begin{array}{l}\text { Eu tento pensar mais sobre os benefícios de comer frutas, legumes } \\
\text { e verduras do que na dificuldade de manter uma alimentação } \\
\text { saudável. }\end{array}$ \\
\hline Q14 & I make back-up plans to be sure I eat fruits \& vegetable everyday. & $\begin{array}{l}\text { Eu tenho planos extras para assegurar que coma frutas, legumes e } \\
\text { verduras todos os dias. }\end{array}$ \\
\hline Q15 & $\begin{array}{l}\text { I try different kinds of fruits \& vegetables so that I have more options to } \\
\text { choose from. }\end{array}$ & $\begin{array}{l}\text { Eu experimento diferentes tipos de frutas, legumes e verduras para } \\
\text { que tenha mais opções de escolha. }\end{array}$ \\
\hline $\mathrm{C} 2$ & Fruits \& vegetables pros \& cons & $\begin{array}{l}\text { Percepção dos facilitadores e percepção das barreiras de frutas, } \\
\text { legumes e verduras }\end{array}$ \\
\hline E2 & $\begin{array}{l}\text { The following statements are different beliefs about eating fruits \& } \\
\text { vegetables. Please rate HOW IMPORTANT each statement is to your } \\
\text { decision to eat } 5 \text { fruits \& vegetables a day. Use the following scale: }\end{array}$ & $\begin{array}{l}\text { As afirmações seguintes representam diferentes crenças sobre } \\
\text { o consumo de frutas, legumes e verduras. Marque QUÃO } \\
\text { IMPORTANTE cada afirmação é para a sua decisão de consumir } \\
\text { cinco porções diárias de frutas, legumes e verduras. Use a escala a } \\
\text { seguir: }\end{array}$ \\
\hline Q16 & I would feel embarrassed if other kids say me eating fruits \& vegetables. & $\begin{array}{l}\text { Eu me sentiria envergonhado se outros estudantes me vissem } \\
\text { comendo frutas, legumes e verduras. }\end{array}$ \\
\hline Q17 & I would have more energy if I ate fruits \& vegetables. & Eu teria mais energia se comesse frutas, legumes e verduras. \\
\hline
\end{tabular}

(continua) 
Tabela 1 (continuação)

\begin{tabular}{|c|c|c|}
\hline \multicolumn{2}{|c|}{ Versão original } & Versão traduzida e adaptada \\
\hline Q18 & $\begin{array}{l}\text { I would be doing something good for my body if I ate fruits \& } \\
\text { vegetables. }\end{array}$ & $\begin{array}{l}\text { Eu estaria fazendo uma coisa boa pelo meu corpo se comesse frutas, } \\
\text { legumes e verduras. }\end{array}$ \\
\hline Q19 & I would need too much help from my parents to eat fruits \& vegetables. & $\begin{array}{l}\text { Eu precisaria de bastante ajuda dos meus pais para comer frutas, } \\
\text { legumes e verduras. }\end{array}$ \\
\hline Q20 & I would feel healthier if I ate fruits \& vegetables. & Eu me sentiria mais saudável se comesse frutas, legumes e verduras. \\
\hline Q21 & It takes too much time to cut up fruits \& vegetables. & Cortar e/ou preparar frutas, legumes e verduras leva tempo demais. \\
\hline Q22 & My parents would be pleased if I ate fruits \& vegetables. & Meus pais ficariam felizes se eu comesse frutas, legumes e verduras. \\
\hline Q23 & I would rather eat sweets or high fat snacks than fruits \& vegetables. & $\begin{array}{l}\text { Eu prefiro comer doces ou lanches calóricos ao invés de frutas, } \\
\text { legumes e verduras. }\end{array}$ \\
\hline Q24 & Eating fruits \& vegetables would be a great way to start the day. & $\begin{array}{l}\text { Comer frutas, legumes e verduras seria uma boa forma de começar } \\
\text { o dia. }\end{array}$ \\
\hline Q25 & Fruits \& vegetables are too difficult to prepare. & Frutas, legumes e verduras são difíceis demais de preparar. \\
\hline C3 & Self-efficacy & Autoeficácia \\
\hline E3 & $\begin{array}{l}\text { There are many things that can get in the way of eating fruits \& } \\
\text { vegetables. Rate HOW SURE you are that you can do the following in } \\
\text { each situation. }\end{array}$ & $\begin{array}{l}\text { Existem muitas coisas que podem dificultar o consumo de frutas, } \\
\text { legumes e verduras. Marque QUÃO SEGURO você está de que } \\
\text { seguiria cada uma das situações abaixo. }\end{array}$ \\
\hline Q26 & Eat 5 servings of fruits \& vegetables everyday? & Comer cinco porções de frutas, legumes e verduras todos os dias? \\
\hline Q27 & Ask someone in your family to buy your favorite fruit or vegetable? & $\begin{array}{l}\text { Pedir a alguém da família para comprar suas frutas, legumes e } \\
\text { verduras favoritos? }\end{array}$ \\
\hline Q28 & Ask for fruits \& vegetables with your lunch? & Pedir para ter frutas, legumes e verduras no almoço? \\
\hline Q29 & Drink $100 \%$ fruit juice instead of fruit punch or soda? & Tomar sucos $100 \%$ naturais ao invés de refrescos ou refrigerantes? \\
\hline Q30 & Eat fruits or vegetables for a snack instead of chips or candy? & $\begin{array}{l}\text { Comer frutas e legumes no lanche em vez de salgadinhos, batata } \\
\text { frita ou doces? }\end{array}$ \\
\hline Q31 & Ask someone in your family to include fruits or vegetables with dinner? & $\begin{array}{l}\text { Pedir para alguém da família incluir frutas, legumes e verduras no } \\
\text { jantar? }\end{array}$ \\
\hline E4 & $\begin{array}{l}\text { During a typical week, how often has a member of your household } \\
\text { (for example, your father, mother, brother, sister, grandparent, or other } \\
\text { relatives) or friends: }\end{array}$ & $\begin{array}{l}\text { Em uma semana típica, com que frequência alguém da sua casa } \\
\text { (por exemplo, seu pai, mãe, irmão, irmã, avós, parentes etc.) } \\
\text { e amigos: }\end{array}$ \\
\hline Q33 & Encouraged you to eat fruits \& vegetables? & Incentivou você a comer frutas, legumes e verduras? \\
\hline Q34 & Told you that you are doing a good job with eating fruits \& vegetables? & Parabenizou você por estar comendo frutas, legumes e verduras? \\
\hline Q35 & Provided fruits \& vegetables as a snack or part of a meal? & $\begin{array}{l}\text { Providenciou frutas, legumes e verduras como lanche ou parte de } \\
\text { uma refeição? }\end{array}$ \\
\hline Q36 & Eaten fruits \& vegetable with you? & Comeu frutas, legumes e verduras com você? \\
\hline Q37 & Do your friends encourage you to eat fruits \& vegetables? & Seus amigos incentivam você a comer frutas, legumes e verduras? \\
\hline Q38 & Do your friends eat fruits \& vegetables with you? & Seus amigos comem frutas, legumes e verduras com você? \\
\hline Q39 & $\begin{array}{l}\text { How many of your five closest friends eat fruits \& vegetables on a } \\
\text { regular basis? }\end{array}$ & $\begin{array}{l}\text { Quantos dos seus cinco amigos mais próximos comem regularmente } \\
\text { frutas, legumes e verduras? }\end{array}$ \\
\hline
\end{tabular}

C: constructo; E: enunciado; Q: questão.

da questão preserva a escala original. Em relação ao fator 2, denominado percepção das barreiras, os resultados da consistência interna, do fator, teste e reteste e a correlação com o estágio de mudança de comportamento foram superiores em relação ao fator 1 .

A escala do construto da autoeficácia apresentou uma boa consistência interna, valores de teste e reteste satisfatórios e correlação signifi- cativa com o estágio de mudança de comportamento. Apesar de a retirada de qualquer fator implicar o aumento da consistência interna, nenhum item foi retirado por apresentar valores acima de 0,67 e para preservar a escala original. Além disso, o aumento do valor de alfa ficaria inferior ao limite do intervalo de confiança.

Por fim, no construto suporte familiar e dos amigos foram identificados dois fatores que, 
Tabela 2

Análise fatorial e estabilidade temporal para o construto de estratégia de mudança para o consumo de frutas, legumes e verduras $(\mathrm{N}=717)$.

\begin{tabular}{|c|c|c|c|c|}
\hline Item & Fator & $\begin{array}{c}\text { Valor de alfa caso fosse } \\
\text { deletado }\end{array}$ & $\mathrm{CCl}$ * & $\begin{array}{c}\text { Correlação com } \\
\text { estratégia de mudança de } \\
\text { comportamento }\end{array}$ \\
\hline Q1 & 0,71 & 0,83 & $0,81(0,69-0,88)$ & $0,51 * \star$ \\
\hline Q2 & 0,54 & 0,83 & $0,69(0,52-0,81)$ & \\
\hline Q3 & 0,74 & 0,83 & $0,65(0,48-0,78)$ & \\
\hline Q4 & 0,72 & 0,83 & $0,86(0,77-0,91)$ & \\
\hline Q5 & 0,66 & 0,83 & $0,75(0,60-0,84)$ & \\
\hline Q6 & 0,78 & 0,83 & $0,64(0,45-0,77)$ & \\
\hline Q7 & 0,79 & 0,83 & $0,81(0,69-0,88)$ & \\
\hline Q8 & 0,62 & 0,82 & $0,68(0,50-0,80)$ & \\
\hline Q9 & 0,74 & 0,83 & $0,84(0,74-0,90)$ & \\
\hline Q10 & 0,75 & 0,83 & $0,73(0,58-0,84)$ & \\
\hline Q11 & 0,79 & 0,83 & $0,74(0,59-0,84)$ & \\
\hline Q12 & 0,77 & 0,83 & $0,79(0,67-0,88)$ & \\
\hline Q13 & 0,74 & 0,83 & $0,78(0,65-0,87)$ & \\
\hline Q14 & 0,74 & 0,83 & $0,69(0,52-0,81)$ & \\
\hline Q15 & 0,72 & 0,83 & $0,80(0,68-0,88)$ & \\
\hline Alfa de Chronbach & & $0,88(0,82-0,93)$ & & \\
\hline Autovalores & & 8,8 & & \\
\hline \% variância explicada & & 59,1 & & \\
\hline $\mathrm{KMO}$ & & 0,95 & & \\
\hline Teste de Bartelett & & $5.866,1(p=0,0001)$ & & \\
\hline
\end{tabular}

$\mathrm{CCl}$ : coeficiente de correlação intraclasse.

* Teste e reteste para estabilidade temporal $(n=53)$;

** $p=0,000$.

juntos, explicaram $63,2 \%$ da variância da escala. Ambos os fatores identificados apresentaram boa consistência interna, valores substanciais de teste e reteste, e correlação significativa com o estágio de mudança de comportamento.

\section{Discussão}

O presente trabalho teve como objetivo traduzir, adaptar e testar as propriedades psicométricas de determinantes psicossociais para o consumo de frutas, legumes e verduras. Os construtos investigados são baseados em teorias de mudança de comportamento 10,11 que foram testadas empiricamente em estudos de validação, transversais e de intervenção na área da nutrição e em áreas correlatas 12,13,14,15,16,17,18,19.

Como ponto positivo pode-se destacar a operacionalização de todas as etapas de equivalência proposta por Reichenheim \& Moraes 21 nesta temática da área de nutrição. Em geral, na saúde pública tem-se observado a inclusão dessas etapas de forma separada 21 . Em adição, no que concerne à equivalência conceitual e de itens, os autores julgam que o instrumento apresenta relevância para a temática. Operacionalmente foram respeitados o layout, as orientações de preenchimento, a sequência das perguntas e as opções de respostas. No modo de aplicação houve um acréscimo de informações baseado no QFA 22 sobre porções de frutas, legumes e verduras. Esse procedimento foi adotado em função da dificuldade de discernimento da população em geral sobre a definição de porções, o que poderia gerar super ou subestimação 26 . Além disso, não foi observada diferença significativa entre o QFA e a medida de frequência do questionário original. É importante ressaltar que grande parte dos itens reflete sentimentos e percepções individuais referentes a frutas, legumes e verduras, não estando diretamente relacionado com a prática cultural de um país específico, o que facilitou este procedimento. 
Tabela 3

Análise fatorial e estabilidade temporal para o construto do processo de tomada de decisão para o consumo de frutas, legumes e verduras $(\mathrm{N}=717)$.

\begin{tabular}{|c|c|c|c|c|c|}
\hline Item & Fator 1 & Fator 2 & $\begin{array}{c}\text { Valor de alfa } \\
\text { caso fosse } \\
\text { deletado }\end{array}$ & $\mathrm{CCl}$ * & $\begin{array}{c}\text { Correlação } \\
\text { com estágio de } \\
\text { mudança de } \\
\text { comportamento }\end{array}$ \\
\hline Q16 & 0,53 & & 0,77 & $0,78(0,66-0,87)$ & $-0,10$ ** \\
\hline Q19 & 0,56 & & 0,75 & $0,69(0,51-0,80)$ & \\
\hline Q21 & 0,55 & & 0,72 & $0,69(0,52-0,81)$ & \\
\hline Q23 & 0,48 & & 0,80 & $0,61(0,42-0,75)$ & \\
\hline Q25 & 0,64 & & 0,73 & $0,55(0,38-0,68)$ & \\
\hline Q17 & & 0,67 & 0,79 & $0,62(0,43-0,76)$ & $0,13 * \star$ \\
\hline Q18 & & 0,68 & 0,77 & $0,74(0,59-0,84)$ & \\
\hline Q20 & & 0,66 & 0,77 & $0,79(0,66-0,87)$ & \\
\hline Q22 & & 0,63 & 0,87 & $0,74(0,60-0,84)$ & \\
\hline Q24 & & 0,59 & 0,80 & $0,67(0,49-0,79)$ & \\
\hline Alfa de Chronbach & $0,77(0,63-0,86)$ & $0,83(0,73-0,90)$ & & & \\
\hline Autovalores & 3,43 & 2,34 & & & \\
\hline \% variância explicada & 34,3 & 23,4 & & & \\
\hline \% variância total & 57,7 & & & & \\
\hline $\mathrm{KMO}$ & 0,83 & & & & \\
\hline Teste de Bartelett & $2.863,1$ & $=0,0001)$ & & & \\
\hline
\end{tabular}

$\mathrm{CCl}$ : coeficiente de correlação intraclasse.

Fator 1: percepção dos facilitadores; fator 2: percepção das barreiras.

* Teste e reteste para estabilidade temporal $(n=53)$;

$\star \star * A=0,000$.

Tabela 4

Análise fatorial e estabilidade temporal para o construto da autoeficácia para o consumo de frutas, legumes e verduras $(\mathrm{N}=717)$.

\begin{tabular}{|c|c|c|c|c|}
\hline Item & Fator & $\begin{array}{l}\text { Valor de alfa caso } \\
\text { fosse deletado }\end{array}$ & $\mathrm{CCl}$ * & $\begin{array}{l}\text { Correlação com } \\
\text { estágio de mudança } \\
\text { de comportamento }\end{array}$ \\
\hline Q26 & 0,70 & 0,87 & $0,77(0,63-0,86)$ & $0,45 * *$ \\
\hline Q27 & 0,77 & 0,86 & $0,68(0,51-0,80)$ & \\
\hline Q28 & 0,84 & 0,84 & $0,63(0,44-0,76)$ & \\
\hline Q29 & 0,71 & 0,86 & $0,78(0,66-0,87)$ & \\
\hline Q30 & 0,77 & 0,85 & $0,65(0,46-0,78)$ & \\
\hline Q31 & 0,82 & 0,85 & $0,70(0,53-0,81)$ & \\
\hline Q32 & 0,67 & 0,87 & $0,74(0,59-0,84)$ & \\
\hline Alfa de Chronbach & & $0,83(0,72-0,90)$ & & \\
\hline Autovalores & & 4,01 & & \\
\hline \% variância & & 57,3 & & \\
\hline \multicolumn{5}{|l|}{ explicada } \\
\hline $\mathrm{KMO}$ & & 0,89 & & \\
\hline Teste de Bartelett & & $2.317,5(p=0,0001)$ & & \\
\hline
\end{tabular}

$\mathrm{CCl}$ : coeficiente de correlação intraclasse.

* Teste e reteste para estabilidade temporal $(n=53)$;

** $p=0,000$. 


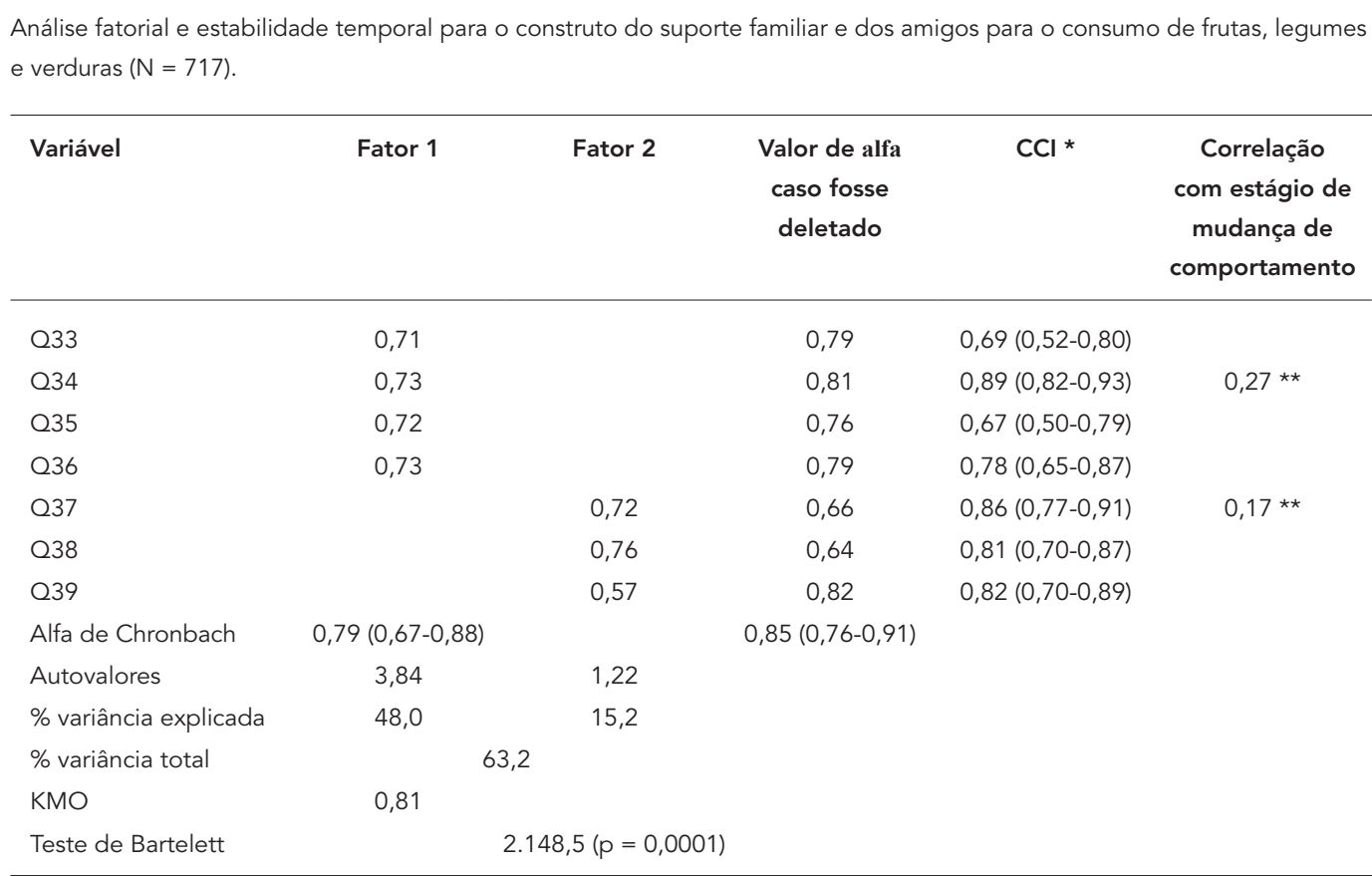

$\mathrm{CCl}$ : coeficiente de correlação intraclasse.

Fator 1: suporte dos pais; fator 2: suporte dos amigos.

* Teste e reteste para estabilidade temporal $(n=53)$;

** $\mathrm{p}=0,000$.

Na equivalência de mensuração, os resultados observados indicam uma boa consistência interna, estabilidade temporal, correlação com os estágios de mudança de comportamento e aplicabilidade em estudantes universitários. Todas as dimensões apresentaram boa consistência interna, com valores de alfa de Cronbach superiores a 0,70 , todos os itens com valores de correlação intraclasse superiores a 0,60 (com $30,7 \%$ acima de 0,80 ) e de correlação com o estágio de mudança de comportamento sempre significativo.

Em comparação com o estudo de Hagler et al. 12 apenas o construto de percepção dos facilitadores apresentou valor de alfa semelhante ( $0,78 v s .0,77$, respectivamente); para a estratégia de mudança de comportamento, autoeficácia, percepção das barreiras e dos facilitadores, e suporte dos pais e amigos os valores encontrados no presente estudo foram sempre superiores. Já em relação ao teste e reteste, os valores encontrados foram semelhantes para os construtos estratégia de mudança de comportamento $(0,74 v$ s. 0,75 , respectivamente) e percepção dos facilitadores $(0,75$ para ambos), mas foi inferior para a autoeficácia $(0,81 v$ s. 0,75 , respectivamente), percepção das barreiras $(0,85 v s$. 0,80 , respectivamente) e suporte dos pais $(0,82 v s .0,75$, respectivamente) e superior para o suporte dos amigos (0,43 vs. 0,83, respectivamente).

Em relação à análise fatorial apenas duas questões, sendo uma no construto de estratégia de mudança de comportamento e outra na percepção de barreiras, apresentaram valores de item abaixo de 0,6. Na questão 2, "Eu estabeleço metas para comer pelo menos cinco porções diárias de frutas, legumes e verduras", o resultado obtido foi 0,54 , o que pode ser reflexo da intenção da amostra, que em parte talvez não esteja interessada na mudança. Já a questão 23, “Eu prefiro comer doces ou lanches calóricos ao invés de frutas, legumes e verduras", foi identificada como fator 1 "percepção dos facilitadores" e teve como valor de item 0,48. Esse resultado pode estar relacionado com a preferência da população estudada por alimentos calóricos ao invés de frutas, legumes e verduras, e assim ser percebida como uma barreira por parte da amostra. Entretanto, ambas as questões tiveram valores acima do recomendado $(0,40)$ e apresentaram boa estabilidade temporal, permanecendo na versão final do instrumento.

Essas diferenças são esperadas, pois quando se planeja a construção de um instrumento para estudos epidemiológicos com pretensões explicativas, esse é composto por diferentes módulos 
que contemplam construtos de um modelo teórico 21 . Vale ressaltar que não existe um modelo teórico hegemônico e que a variabilidade dos modelos depende dos procedimentos utilizados para a validação. Nesse sentido, as adaptações tendem a incorporar os itens de forma que apresentem maior aplicabilidade e propriedades psicométricas satisfatórias em uma população específica.

Algumas limitações devem ser consideradas para a extrapolação dos resultados encontrados. $\mathrm{O}$ instrumento foi testado em uma amostra de adultos jovens universitários de Recife (Pernambuco). Considerando que no Brasil existem importantes diferenças culturais e socioeconômicas, outros trabalhos devem considerar tam-

\section{Resumen}

El objetivo fue traducir, adaptar y probar las propiedades psicométricas de mediadores psicosociales para el consumo de frutas y verduras. Un cuestionario basado en los constructos multidimensionales de la Teoría Cognitiva Social y el modelo transteórico se administró a 717 estudiantes universitarios. Los procedimientos de validación fueron: equivalencia conceptual, artículo, semántica, operativos, de medición y funcional. El análisis factorial exploratorio y la estabilidad temporal se realizaron con SPSS (17.0). Se observaron pequeñas diferencias entre las versiones traducidas y se hicieron algunas adaptaciones. Se observó la varianza explicada entre $57,3 \%$ y $63,2 \%$ y los valores de $\alpha$ osciló desde 0,77 hasta 0,88, que muestra una buena consistencia interna. La estabilidad temporal media era buena y todos los constructos se correlacionaron con la etapa de cambio de comportamiento $(p<0,05)$. Todos los pasos de validación fueron considerados satisfactorios y adecuados para aplicación en la población. bém essas características. No que tange ao modo de aplicação, apesar de não haver diferença significativa entre o QFA e o instrumento original, em ambos existe a necessidade de esclarecimento relacionado a porções. Esse procedimento torna a aplicação mais extensa, porém reduz o viés de informação. Particularmente, os resultados encontrados permitem a utilização do instrumento tanto em estudos com o objetivo de investigar determinantes psicossociais para a mudança de comportamento, bem como para auxiliar proposições de intervenções. Sugere-se para trabalhos futuros a utilização da análise fatorial confirmatória para a testagem da validade convergente e dos fatores que compõem o instrumento.

\section{Colaboradores}

R. M. Tassitano participou da concepção e projeto, análise e interpretação dos dados, redação do artigo e aprovação final da versão a ser publicada. P. C. Cabral contribuiu na concepção do projeto, revisão crítica relevante do conteúdo intelectual e aprovação final da versão a ser publicada. G. A. P. Silva colaborou na concepção do projeto, interpretação dos dados, revisão crítica intelectual e aprovação final da versão a ser publicada.

\section{Agradecimentos}

À Universidade Federal Rural de Pernambuco (UFRPE) pelo apoio logístico. 


\section{Referências}

1. Ness AR, Powles JW. Fruit and vegetable and cardiovascular disease: a review. Int J Epidemiol 1997; 26:1-13.

2. World Cancer Research Fund; American Institute for Cancer Research. Food, nutrition and the prevention of cancer: a global perspective. Washington DC: American Institute for Cancer Research; 1997.

3. World Health Organization. Global health risks: mortality and burden of disease attributable to selected major risks. Geneva: World Health Organization; 2009.

4. Glanz K, Rimer BK, Viswanath K. Health behavior and health education. Theory, research, and practice. $4^{\text {th }}$ Ed. San Francisco: John Wiley \& Sons; 2008.

5. Ammerman AS, Lindquist $\mathrm{CH}$, Lohr KN, Hersey J. The efficacy of behavioral interventions to modify dietary fat and fruit and vegetable intake: a review of the evidence. Prev Med 2002; 35:25-41.

6. Shaikh AR, Yaroch AL, Nebeling L, Yeh M-C, Resnicow K. Psychosocial predictors of fruit and vegetable consumption in adults: a review of the literature. Am J Prev Med 2008; 34:535-43.

7. Guillaumie L, Godin G, Vézina-Im LD. Psychosocial determinants of fruit and vegetable intake in adult population: a systematic review. Int J Behav Nutr Phys Act 2010; 2:7-12.

8. Brug J, Oenema A, Ferreira I. Theory, evidence and intervention mapping to improve behavior nutritional and physical activity interventions. Int J Behav Nutr Phys Act 2005; 2:1-7.

9. Pormerleau J, Lock K, Knai C, McKee M. Interventions designed to increase adult fruit and vegetable intake be effective: a systematic review of the literature. J Nutr 2005; 135:2486-95.

10. Bandura A. Human agency in social cognitive theory. Am Psychol 1989; 44:1175-84.

11. Prochaska JO, Velicer WF. The Transtheoretical Model of health behavior change. Am J Health Promot 1997; 12:38-48.

12. Hagler AS, Norman GJ, Radick LR, Calfas KJ, Sallis JF. Comparability and reliability of paper and computer based measures of psychosocial constructors for adolescent fruit and vegetable and dietary fat intake. J Am Diet Assoc 2005; 105:1758-64.

13. Zabinski MF, Daly T, Norman GJ, Rupp J, Calfas KJ, Sallis JF, et al. Psychosocial influences on fruit, vegetable and dietary fat intake among adolescent boys and girls. J Am Diet Assoc 2006; 106:814-21.

14. Norman GJ, Sallis JS, Gaskins R. Comparability and reability of paper- and computer-based measures of psychosocial constructs for adolescent physical activity and sedentary behavior. Res Q Exerc Sport 2005; 76:315-23.

15. Hagler AS, Calfas KJ, Norman GJ, Sallis JF, Patrick K. Construct validity of physical activity and sedentary behaviors staging measures for adolescents. Ann Behav Med 2006; 31:186-93.
16. Patrick K, Calfas KJ, Norman GJ, Zabinski MF, Sallis JF, Rupp J, et al. Randomized controlled trial of a primary care and home-based intervention for physical activity and nutrition behaviors: PACE+ for adolescents. Arch Pediatr Adolesc Med 2006; 160:128-36.

17. Calfas KJ, Sallis JF, Zabinski MF, Wilfley DE, Rupp J, Prochaska JJ, et al. Preliminary evaluation of a multicomponent program for nutrition and physical activity change in primary care: PACE+ for adults. Prev Med 2002; 34:153-61.

18. Patrick K, Sallis JF, Prochaska JJ, Lydston DD, Calfas KJ, Zabinski MF, et al. A multicomponent program for nutrition and physical activity change in primary care: PACE+ for adolescents. Arch Pediatr Adolesc Med 2001; 155:940-6.

19. Carlson JA, Sallis JF, Ramirez ER, Patrick K, Norman GJ. Physical activity and dietary behavior change in Internet-based weight loss interventions: comparing two multiple-behavior change indices. Prev Med 2012; 54:50-4.

20. Dixon JK. Factor analysis. In: Munro BH, editor. Statistical methods for health care research. $4^{\text {th }}$ Ed. New York: Lippincott; 2001. p. 303-29.

21. Reichenheim ME, Moraes CL. Operacionalização de adaptação transcultural de instrumentos de aferição usados em epidemiologia. Rev Saúde Pública 2007; 41:665-73.

22. Slater B, Philippi ST, Fisberg RM, Latorre MR. Validation of semi-quantitative adolescent food frequency questionnaire applied at a public school in São Paulo, Brazil. Eur J Clin Nutr 2003; 57:629-35.

23. Painter J, Rah JH, Lee YK. Comparison of international food guide pictorial representations. J Am Diet Assoc 2002; 102:483-9.

24. Kloek GC, van Lenthe FJ, van Nierop PW, Mackenbach JP. Stages of change for fruit and vegetable consumption in deprived neighborhoods. Health Educ Behav 2004; 31:223-41.

25. Landis JR, Koch GG. The measurement of observer agreement for categorical data. Biometrics 1977; 33:159-74.

26. Kim DJ, Holowaty EJ. Brief, validated survey instruments for the measurement of fruit and vegetable intakes in adults: a review. Prev Med 2003; 36:440-7.

Recebido em 19/Out/2012

Versão final reapresentada em 25/Jun/2013

Aprovado em 14/Ago/2013 